\title{
La concurrence entre savoirs scientifiques et croyances religieuses à l'école
}

Enquête auprès d'élèves de terminale en Belgique francophone et pistes pédagogiques

The competition between scientific knowledge and religious beliefs at school. Survey of final-year secondary students in French-speaking Belgium and avenues for teaching

La competencia entre saberes científicos y creencias religiosas en la escuela. Encuesta con unos alumnos de bachillerato en la Bélgica francófona y pistas pedagógicas

José-Luis Wolfs

\section{CpenEdition}

Journals

Édition électronique

URL : https://journals.openedition.org/ries/6146

DOI : 10.4000/ries.6146

ISSN : 2261-4265

Éditeur

France Education international

Édition imprimée

Date de publication : 30 avril 2018

Pagination : 103-112

ISBN : 978-2-85420-618-0

ISSN : $1254-4590$

\section{Référence électronique}

José-Luis Wolfs, "La concurrence entre savoirs scientifiques et croyances religieuses à l'école »,

Revue internationale d'éducation de Sèvres [En ligne], 77 | avril 2018, mis en ligne le 30 avril 2020, consulté le 24 juin 2021. URL : http://journals.openedition.org/ries/6146 ; DOI : https://doi.org/ $10.4000 /$ ries.6146 


\title{
La concurrence entre savoirs scientifiques et croyances religieuses à l'école
}

\section{Enquête auprès d'élèves de terminale en Belgique francophone et pistes pédagogiques}

\author{
José-Luis Wolfs \\ Université libre de Bruxelles
}

Cet article traite des conflits possibles entre savoirs scientifiques et croyances religieuses à l'école. La commémoration du $150^{\mathrm{e}}$ anniversaire de la publication de l'œuvre de Darwin, en 2009, a été l'occasion d'une prise de conscience du rejet total ou partiel dont la théorie de l'évolution faisait encore l'objet, de la part d'une partie des élèves et des enseignants (Clément, 2014), dans différentes parties du monde, y compris en Europe.

Notre préoccupation plus générale, en amont de la question spécifique de l'acceptation ou non de la théorie de l'évolution, est celle de savoir dans quelle mesure les élèves, au terme de l'enseignement secondaire, ont acquis ou non une conception "sécularisée » de la science. Il s'agit là, en effet, d'un enjeu fondamental, tant sur le plan épistémologique et sociétal qu'éducatif, dans la mesure où il importe de garantir à la fois la liberté de conscience et de croyance de chacun et chacune et la nécessaire autonomie du registre explicatif scientifique à l'égard des croyances religieuses.

Dans cet article, nous commencerons par exposer différents positionnements théoriquement possibles entre sciences et croyances religieuses. Ensuite, sur la base de cette grille de lecture, nous examinerons dans quelle mesure des élèves de terminale en Belgique francophone, de différentes convictions (catholiques, musulmans, protestants, agnostiques-athées) et filières d'études, ont acquis ou non une conception sécularisée de la science. Nous terminerons enfin par quelques pistes de réflexion sur le plan pédagogique et didactique.

\section{LES POSITIONNEMENTS POSSIBLES ENTRE SCIENCES ET CROYANCES RELIGIEUSES}

À partir de différentes études à caractère historique et sociologique (Minois, 1990 ; Urvoy, 2006), de grilles de lecture existantes (Rasi, 2003 ; Lambert, 1999) et d'enquêtes de terrain, un modèle visant à prendre en compte différents positionnements théoriquement possibles entre sciences et croyances religieuses a été élaboré (Wolfs, 2013). 
Il comprend sept idéaux-types contrastés, dont quatre conduisent à des situations de conflit, de confusion ou d'amalgame entre science et religions et trois, au contraire, à une délimitation de leurs régimes respectifs et à différents modes de cohabitation.

(1) Le rejet total ou partiel de contenus scientifiques au nom de conceptions de type « fidéiste » (primauté de la foi sur la raison), s'appuyant par exemple sur une interprétation littérale des Écritures.

(2) Le concordisme dit «classique». Le concordisme repose globalement sur le postulat selon lequel le «livre de la Parole» (par exemple, la Bible ou le Coran) et le «livre de la Nature $»^{1}$, que s'efforcent de déchiffrer les sciences, ne sauraient se contredire, puisque tous deux trouvent leur origine en Dieu. La tentation peut dès lors être grande - c'est ce que nous appelons le concordisme sous sa forme "classique »- de vouloir lire le «livre de la Nature " en fonction des catégories conceptuelles du « livre de la Parole», dans le but, en particulier, de chercher à «confirmer " par la science ce que les Écritures auraient révélé, ou plus largement, d'établir une forme d'alliance entre sciences et Écritures (ou théologie), sous l'autorité de ces dernières. Il s'agit, par exemple, d'associer les jours du récit de la Genèse aux différentes ères géologiques ou encore l'image de montagnes se déplaçant (évoquées dans un verset du Coran) et la tectonique des plaques.

(3) Le concordisme dit "inversé » : celui-ci vise à établir des concordances entre sciences et croyances religieuses (ou plus largement métaphysiques), en partant non pas des Écritures ou d'une tradition révélée, comme dans le cas du concordisme classique, mais d'une démarche qui se présente comme «scientifique ». Il s'agit en quelque sorte de vouloir trouver Dieu, défini éventuellement sous la forme d'un principe abstrait ou d'un grand architecte, à travers la «science ", quitte à créer une pseudo-science pour tenter d'y parvenir. C'est le cas en particulier aujourd'hui des tenants du «dessein intelligent» (intelligent design).

(4) La recherche d'une complémentarité entre science et religion, sous des formes autres que concordistes, basée sur la reconnaissance de la différence de nature fondamentale entre les deux registres et un respect clair de l'autonomie de la science.

(5) Nous n'avons pas à chercher dans l'Écriture un enseignement proprement dit de l'astronomie (...) et l'intention du Saint-Esprit est de nous enseigner comment l'on doit aller au ciel et non comment va le ciel...2 (Galilée).

Cette idée, déjà énoncée par Galilée, a été reprise notamment par S. Gould (2000) sous l'acronyme «Noma » (non overlapping magisteria). Dans

1. La Nature peut être définie métaphoriquement comme étant un « livre » à découvrir ou à comprendre.

2. Montrant ainsi que science et religion remplissent des fonctions différentes dans la vie d'une personne croyante. 
une perspective sensiblement différente, Lambert (1999) désigne, sous le vocable " articulation ", des essais de mise en relation indirecte entre science et théologie, passant par la médiation d'approches philosophiques telles que l'éthique, l'herméneutique ou la métaphysique et évitant en principe toute forme de rapprochement direct entre un passage des Écritures et un énoncé scientifique.

(6) Le principe d'autonomie de la démarche scientifique à l'égard des croyances religieuses, sans recherche de complémentarité entre science et religion. Les postures 4 et 5 se fondent sur un principe commun, qui est celui de l'autonomie de la science à l'égard des croyances religieuses. Celui-ci est le fruit d'une conquête de plusieurs siècles de la part de savants tant croyants que non croyants. Il repose sur le postulat de travail suivant: dans la démarche scientifique, la nature s'explique par la nature (et non par le livre de la Parole). On quitte dès lors le domaine de la science chaque fois que l'on invoque des facteurs surnaturels. La science vise en effet à construire des représentations du monde, en veillant à respecter certaines règles méthodologiques spécifiques, qui se sont précisées ou reformulées au cours du temps : le "principe de parcimonie » dans la démarche explicative (énoncé par Guillaume d'Ockham au XIVe siècle), la limitation des ambitions de la science à la recherche des "causes efficientes » et non à celle des "causes ultimes ", proposée par Descartes et Galilée au XVII ${ }^{\mathrm{e}}$ siècle, le principe de "réfutabilité », énoncé par Popper au $\mathrm{XX}^{\mathrm{e}}$ siècle. Ces trois caractéristiques permettent de baliser plus précisément les questions qui relèvent ou au contraire ne relèvent pas de la science. ${ }^{3}$

(7) La critique rationaliste, au nom de la science, de conceptions religieuses (forme non scientiste). Cette posture se fonde notamment sur le principe selon lequel la démarche scientifique s'est construite par ruptures et dépassements successifs par rapport à un premier niveau d'explication mythologique ou religieux développé par l'être humain face à l'univers. Certaines croyances religieuses particulières ou certaines formes d'intolérance de la part d'autorités religieuses peuvent être perçues comme des freins ou des obstacles au développement de la science, voire à l'émancipation de l'humanité. Cette conception doit être clairement distinguée de la suivante.

(8) La critique rationaliste, de forme scientiste, où la science est conçue comme un registre de pensée totalisant et excluant par rapport à d'autres, notamment religieux (cas extrême : les persécutions développées par le régime de l'ex URSS à l'égard des religions, ainsi qu'à l'égard de la pensée scientifique non conforme à celle de Lyssenko).

3. Ainsi, si l'on admet ces critères, les questions qui touchent à l'existence ou à la non-existence de Dieu ne font pas partie du champ de la science et ce par choix méthodologique et non de conviction. Ces questions portent en effet sur les « causes ultimes », elles ne sont pas « réfutables » (au sens de Popper), etc. 
La figure suivante permet de mieux visualiser ces différentes conceptions, ainsi que les critères distinctifs sous-jacents.

Types de positionnements possibles entre sciences et croyances religieuses ${ }^{4}$

\begin{tabular}{|c|c|c|c|c|c|c|}
\hline Posture 1 & Posture 2 & Posture 3 & Posture 4 & Posture 5 & Posture 6 & Posture 7 \\
\hline \multirow{2}{*}{$\begin{array}{l}\text { Rejet } \\
\text { de la science, } \\
\text { au nom de } \\
\text { conceptions } \\
\text { religieuses de } \\
\text { type fidéiste }\end{array}$} & \multirow[t]{2}{*}{$\begin{array}{l}\text { Concordisme } \\
\text { classique }\end{array}$} & \multirow[t]{2}{*}{$\begin{array}{l}\text { Concordisme } \\
\text { inversé }\end{array}$} & \multicolumn{2}{|c|}{$\begin{array}{l}\text { Autonomie mutuelle } \\
\text { entre science } \\
\text { et croyances religieuses }\end{array}$} & \multicolumn{2}{|c|}{$\begin{array}{l}\text { Critiques rationalistes, } \\
\text { au nom de la science, } \\
\text { de conceptions religieuses }\end{array}$} \\
\hline & & & $\begin{array}{l}\text { Avec } \\
\text { recherche } \\
\text { d'une forme } \\
\text { de complé- } \\
\text { mentarité } \\
\text { non- } \\
\text { concordiste }\end{array}$ & $\begin{array}{l}\text { Sans } \\
\text { recherche } \\
\text { de complé- } \\
\text { mentarité }\end{array}$ & $\begin{array}{l}\text { Forme non- } \\
\text { scientiste }\end{array}$ & $\begin{array}{l}\text { Forme } \\
\text { scientiste }\end{array}$ \\
\hline \multicolumn{3}{|c|}{ Prééminence de croyances religieuses } & \multicolumn{2}{|c|}{ Absence de prééminence } & \multicolumn{2}{|c|}{ Prééminence de la science } \\
\hline $\begin{array}{l}\text { Pas de } \\
\text { recherche } \\
\text { «d'alliance» }\end{array}$ & \multicolumn{3}{|c|}{$\begin{array}{l}\text { Recherche «d'alliance » science - religion } \\
\text { selon des perspectives très différentes }\end{array}$} & \multicolumn{3}{|c|}{$\begin{array}{l}\text { Pas de recherche « d'alliance» } \\
\text { science - religion }\end{array}$} \\
\hline \multicolumn{3}{|c|}{ Confusion science - religion } & \multicolumn{3}{|c|}{$\begin{array}{l}\text { Reconnaissance de la spécificité du registre } \\
\text { explicatif scientifique et de son autonomie } \\
\text { par rapport aux croyances religieuses }\end{array}$} & $\begin{array}{l}\text { Confusion } \\
\text { science - } \\
\text { religion }\end{array}$ \\
\hline \multicolumn{3}{|c|}{ Conceptions non sécularisées de la science } & \multicolumn{3}{|c|}{ Conceptions sécularisées de la science } & $\begin{array}{l}\text { Sécularisme } \\
\text { excluant }\end{array}$ \\
\hline
\end{tabular}

Il est important de souligner qu'il s'agit d'une typologie de postures et non d'individus. Une même personne peut éventuellement adhérer à plusieurs de ces postures, selon les matières concernées ou les questions posées.

Les trois premières conceptions peuvent être qualifiées de non sécularisées, dans la mesure où la science est, à des degrés divers, sous le contrôle du religieux et qu'elle n'est pas autonome ou indépendante par rapport à lui. Dans les quatre conceptions suivantes, la science est autonome et indépendante par rapport à celui-ci. On peut parler de conceptions «sécularisées » dans trois cas et d'un "sécularisme » de type excluant pour le quatrième (scientisme), dans la mesure où il ne s'agit pas seulement d'une indépendance à l'égard du religieux mais d'une volonté d'éradication de celui-ci.

4. Ce modèle a été construit en articulant une démarche de type déductif, qui a consisté à définir des catégories conceptuelles abstraites à partir de deux critères généraux (la prééminence ou l'absence de prééminence entre sciences et croyances religieuses, la recherche ou non d'une forme de complémentarité entre les deux), à une démarche inductive, qui a consisté à analyser un grand nombre de prises de position différentes issues de contextes historiques et culturels variés (à partir d'ouvrages historiques, d'enquêtes de terrain, etc.). 


\section{DANS QUELLE MESURE LES ÉLĖVES DE TERMINALE ONT-ILS UNE CONCEPTION SÉCULARISÉE OU NON DE LA SCIENCE ?}

Un questionnaire comportant une trentaine de questions de type fermé et couvrant les différents idéaux-types du modèle de référence (fidéisme, concordisme classique, concordisme inversé, autonomie de la science, complémentarité et critiques rationalistes $)^{5}$ a été administré, en Belgique francophone, à des élèves de terminale : 414 musulmans, 202 protestants ${ }^{6}, 344$ catholiques et 440 agnostiques ou athées, de trois filières d'études : technique ou professionnelle (TP), générale sciences de base (GSB), générale sciences fortes (GSF) ${ }^{7}$. Les élèves musulmans et protestants ont été volontairement surreprésentés afin de rendre possible des comparaisons. Pour caractériser une conception de la science de " sécularisée ", deux indicateurs ont été définis : le rejet des conceptions fidéistes et concordistes (sous forme classique et inversée) et l'adhésion à l'idée d'autonomie de la science à l'égard des croyances religieuses.

Premier constat : l'adhésion à une conception sécularisée de la science, au sens défini ci-dessus, apparaît fortement liée aux convictions personnelles des élèves. Ainsi, au sein de cet échantillon, une majorité d'élèves catholiques (61\%) a une conception sécularisée de la science, alors que ce n'est le cas que chez $21 \%$ des protestants ou $13 \%$ des musulmans.

Deuxième constat : l'effet de la filière d'études diffère fortement selon les convictions personnelles des élèves. Ainsi, chez les élèves musulmans, la proportion d'élèves ayant une conception sécularisée de la science est très faible dans les trois filières d'études $(8 \%, 16 \%$ et $14 \%)$. Chez les élèves protestants, ces proportions sont de $16 \%, 19 \%$ et $29 \%$. Dans ces deux cas, aucun effet statistiquement significatif de la filière d'études ne peut être mis en évidence. Chez les élèves catholiques, un effet statistiquement significatif ${ }^{8}$ de la filière générale d'études peut être observé, avec un taux de $41 \%$ en technique professionnelle contre $67 \%$ et $74 \%$ dans les filières générales, mais qui doit être interprété avec prudence, compte tenu en particulier des niveaux d'étude sensiblement différents des parents entre ces filières. On peut remarquer aussi, chez les élèves agnostiques ou athées, qu'une conception sécularisée de la science ne va pas automatiquement de soi, en particulier dans la filière technique-professionnelle,

5. Il est important de préciser qu'aucune question ne portait sur la théorie de l'évolution, afin d'examiner les conceptions sécularisées ou non sécularisées de la science chez ces élèves, en dehors de ce sujet spécifique.

6. Ceux-ci sont principalement issus des mouvances évangéliques, pentecôtistes, etc., d'origines culturelles très diverses, mais principalement d'Afrique centrale.

7. Les élèves en « sciences fortes » ont au moins six heures par semaine de physique, chimie, biologie, contre trois heures en " sciences de base ».

8. $\mathrm{Chi}^{2}(2 \mathrm{dl})=29,6$, S.000. 
où ce taux est de $65 \%$ contre $84 \%$ et $93 \%$ dans les filières générales ${ }^{9}$. Des difficultés de compréhension des questions et/ou une faible culture à la fois en matière scientifique et à propos des questions religieuses pourrait expliquer ce résultat quelque peu paradoxal.

Troisième constat : le rapport aux Écritures sacrées varie très fortement, au sein de cet échantillon, selon les religions considérées. La proportion d'élèves attribuant à celles-ci le statut de "récit réaliste » ${ }^{10}$ (plutôt que "symbolique » et/ou " mythique») est de $74 \%$ chez les élèves musulmans, $57 \%$ chez les protestants et $20 \%$ chez catholiques.

Quatrième constat : la probabilité, pour les élèves croyants, d'adopter une conception sécularisée de la science tend à être plus élevée s'ils attribuent aux Écritures sacrées le statut de "récit symbolique et/ou mythique " plutôt que celui de "récit réaliste »: $30 \%$ contre $8 \%$ chez les musulmans, $33 \%$ contre $12 \%$ chez les protestants et $66 \%$ contre $36 \%$ chez les catholiques. La différence est chaque fois statistiquement significative ${ }^{11}$. On constate néanmoins que ce facteur n'a qu'un effet limité.

Les différences importantes observées entre élèves de religions différentes, à la fois en termes de rapport aux Écritures sacrées ou d'adoption d'une conception sécularisée de la science, renvoient à des facteurs explicatifs nombreux (historiques, sociologiques, cultuels, religieux) qui mériteraient de faire l'objet d'une analyse beaucoup plus approfondie dépassant le cadre de cet article. Le rapport à la sécularisation a en effet été très différent à la fois selon les religions et selon les contextes historiques ou culturels considérés. Ajoutons aussi qu'en cas de crispation identitaire, dans un contexte de dévalorisation perçue de leurs croyances ou de leurs caractéristiques culturelles, ce qui est parfois le cas dans le cadre de l'immigration, des élèves pourraient, par un processus de survalorisation ontologique (Heine et al., 2008) et/ou d'(auto)-assignation identitaire (Mathieu, 2011), faire passer au premier plan une composante de leur identité qu'ils jugeraient essentielle, par exemple leurs croyances religieuses et certaines conceptions non sécularisées qui pourraient leur être associées.

Dès lors, comme nous le notions (Wolfs et Delhaye, 2016), il pourrait y avoir, pour certains, un cumul de facteurs conduisant à une conception peu sécularisée de la science : s’identifier à une obédience religieuse historiquement et sociologiquement peu sécularisée (par exemple, être un élève protestant évangélique), être originaire d'une société elle aussi globalement peu sécularisée et enfin, dans un contexte d'immigration, avoir éventuellement développé des stratégies identitaires de survalorisation ontologique, mettant au premier plan certaines traditions ou pratiques religieuses peu sécularisées, par exemple une lecture littérale des Écritures.

\footnotetext{
9. Cette différence est aussi statistiquement significative $\left(\mathrm{Chi}^{2}(2 \mathrm{dl})=35, \mathrm{~S} .000\right)$.

10. Expliquant réellement et matériellement l'origine de l'univers et l'origine de l'homme.

11. $\mathrm{Chi}^{2}(1 \mathrm{dl})=27,5$, S.ooo pour les musulmans, $\mathrm{Chi}^{2}(1 \mathrm{dl})=11,8$, S.001 pour les protestants, $\mathrm{Chi}^{2}(1 \mathrm{dl})=20$, S.000 pour les catholiques.
} 


\section{QUELQUeS PISTES DE RÉFLEXION SUR LE PLAN PÉDAGOGIQUE ET DIDACTIQUE}

Cette enquête a mis en évidence la non acquisition d'une conception sécularisée de la science, chez une partie importante des élèves, au terme de l'enseignement secondaire. Face à ce constat, plusieurs pistes d'actions sont envisageables, sur le plan pédagogique et didactique, afin de leur permettre de mieux distinguer les deux registres de discours et de mieux comprendre les enjeux d'une conception sécularisée de la science.

Une première piste vise à donner aux élèves une connaissance mieux informée de l'objet et des limites de la science (Aroua et al., 2012). Elle peut s'effectuer en accompagnant l'enseignement des sciences d'une réflexion épistémologique explicite, insistant en particulier sur les choix méthodologiques suivants : la volonté d'expliquer la nature par la nature, le souci de la preuve et de la démonstration, la réfutabilité (Popper), la parcimonie (G. d'Ockham), la recherche des causes efficientes plutôt que des causes ultimes (Descartes, Galilée), etc. Pour comprendre la nature de la science, il est très important de s'intéresser à la manière dont celle-ci se construit. Quelques éléments d'histoire des sciences sont donc indispensables. C'est l'occasion aussi de souligner et de valoriser, auprès des élèves, l'apport de différentes cultures au développement de la science. $^{12}$

Une seconde piste, dans un autre registre qui peut être celui d'un enseignement des faits religieux, pourrait avoir pour but de faire prendre conscience aux élèves de la diversité des lectures dont les Écritures sacrées peuvent faire font l'objet, selon les contextes historiques et culturels, afin notamment que ceux et celles qui seraient tenté(e)s par une lecture de type littéraliste, puissent s'en distancier. Le statut attribué aux Écritures sacrées constitue en effet une des variables clés dans l'adoption d'une conception sécularisée ou au contraire non sécularisée de la science.

Ces deux premières pistes sont-elles suffisantes pour permettre aux élèves d'éviter toute forme de confusion ou d'amalgame ente science et croyance religieuse ? Il semblerait que même après avoir pris du recul par rapport à leurs conceptions initiales en matière de science et/ou de religion, certains élèves restent néanmoins assez perplexes, lorsqu'il s'agit d'expliciter les positionnements possibles entre les deux. La grille de lecture présentée au point 2, comme outil de recherche, peut dès lors assez aisément être adaptée en outil de formation. Cette troisième piste permet ainsi aux élèves d'envisager plusieurs options possibles et de les argumenter.

12. À titre d'exemple, une exposition itinérante, organisée en Belgique francophone en 2007 sur le thème "À la découverte de l'âge d'or des sciences arabes », a permis à de nombreuses classes de prendre conscience de la richesse de cet apport et de renforcer les liens d'estime et de respect entre cultures différentes. 
Science et religion ne sont toutefois pas les deux seuls types de discours qu'il convient d'apprendre à différencier. Une réflexion plus large sur les caractéristiques de différents discours mérite aussi d'être envisagée. À titre d'illustration de cette quatrième piste, mentionnons un dispositif mis en place par une enseignante de français. L'établissement, situé au centre de Bruxelles, accueille des élèves de quarante origines nationales différentes, issus surtout de l'immigration maghrébine et africaine. Tous ces élèves se déclarent croyants (musulmans, protestants, catholiques). Le but du dispositif est d'apprendre aux élèves à distinguer plusieurs types de discours (religieux ou métaphysiques, philosophiques, scientifiques, etc.) et d'apprendre à penser de manière plus complexe, autonome et critique, en passant notamment du «nous» au « je».

Concrètement, les élèves doivent réaliser un "projet » consistant à examiner une question à partir de deux référentiels différents, à choisir parmi ceux proposés, afin de faire ressortir la spécificité de chacun d'eux. Les questions, servant de support à ces projets, sont choisies par les élèves en concertation avec le professeur. En voici quelques-unes, à titre d'illustration :

- La science a-t-elle réponse à tout?

- Le capitalisme est-il une religion moderne?

- Le darwinisme et le créationnisme sont-ils inconciliables?

- Comment concevoir l'infini ?

- D'où venons-nous? L'origine de l'univers.

- Existe-t-il une vérité absolue ou n'est-elle que relative?

Les témoignages d'élèves recueillis en fin d'année à propos de ce projet sont plutôt encourageants, en particulier dans le contexte actuel, où différentes formes d'extrémisme ou de fondamentalisme sont présentes. Ils tendent à montrer que ces élèves, tous croyants, sont arrivés à prendre du recul par rapport à leurs croyances (en évitant de leur donner le statut de discours absolu et totalisant) et à mieux distinguer différents registres de discours. En voici quelques extraits (F. Meurant, communication personnelle, 11 février 2015):

Mohamed : "Ce travail m'a permis de comprendre qu'il est impossible de construire une nation ou une société dans laquelle il existe une seule vérité absolue, car elle entraîne toujours un dogme, un système totalitaire où le droit à la connaissance est contrôlé par certaines personnes qui vous imposent leurs idées et que vous devez accepter comme vraies, au risque d'être pourchassé, voire exécuté comme ce fut le cas pour Giordano Bruno.»

Nono : «Finalement, il existe une grande différence entre la croyance et le savoir, l'un et l'autre occupent leur domaine propre. Je peux croire en Dieu et garder cette croyance pour moi, tout en étant un scientifique qui conçoit la vérité comme vraie jusqu'à preuve du contraire.»

Azzedine : «L'homme peut prétendre détenir la vérité s'il admet qu'elle est vraie pour lui mais qu'il n'essaie pas de l'imposer aux autres. Pour moi, il n'y a pas de vérité absolue, puisqu'elle est constamment mise à l'épreuve du temps, de l'expérience, de l'évolution du monde. » 
Ajoutons également que les questions d'attitudes à l'égard de la science et/ou des religions ne dépendent pas que de facteurs strictement rationnels, mais aussi émotionnels et affectifs. Des élèves qui se sentiraient dévalorisés, voire discriminés au sein de l'école ou de la société pourraient, en retour, rejeter des contenus scolaires qu'ils jugeraient menaçants pour leur identité. Il convient de créer un climat propice basé sur la sécurité affective, le respect, la reconnaissance, etc.

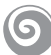

Dans le cadre général du thème "les conflits de vérité à l'école », objet du dossier 77 de la Revue internationale d'éducation de Sèvres, le but de cet article était d'examiner plus précisément les conflits pouvant exister entre sciences et croyances religieuses à l'école. À cette fin, un cadre d'analyse permettant de mieux comprendre les positionnements possibles entre sciences et croyances religieuses a été proposé. À partir de celui-ci, un état des lieux a été esquissé en Belgique francophone auprès d'un échantillon de 1400 élèves de terminale. Il apparaît qu'une proportion non négligeable d'élèves, au terme de l'enseignement secondaire, n'a pas intégré une conception sécularisée de la science, ouvrant ainsi la voie à différentes formes de confusions, amalgames ou instrumentalisations possibles entre sciences et croyances religieuses. Sur la base de ce constat, plusieurs pistes de réflexion et d'action sur le plan pédagogique et didactique ont été suggérées, afin d'amener les élèves à mieux différencier les deux registres.

Il conviendrait bien sûr, en matière de recherche, d'examiner plus en profondeur les facteurs explicatifs des constats effectués et de tester plus systématiquement les dispositifs didactiques suggérés. Sur un plan pédagogique concret se pose aussi la question du cadre institutionnel où traiter ces thèmes avec les élèves : cours de sciences, de philosophie, d'histoire, enseignement des faits religieux, français, éducation aux médias, etc. ? Les enjeux en présence sont suffisamment importants pour qu'un cadre clair et précis puisse être défini et mis en œuvre, en concertation avec l'ensemble des acteurs éducatifs concernés.

\section{BIBLIOGRAPHIE}

AROUA S, COQUIDE S., ABBES S. (2012) : "Controverses dans l'enseignement de l'évolution. Questions de recherche sur les stratégies d'intervention en classe et dans la formation ", Revue des Sciences et des Technologies, $\mathrm{n}^{\circ}$ 5, p. 47-76.

CLEMENT P. (2014) : «Les conceptions créationnistes d'enseignants varient-elles en fonction de leur religion?", Éducation et Sociétés, n 33, p. 113-136.

GOULD J. (2000) : Et Dieu dit que Darwin soit!, Paris, Seuil.

LAMBERT D. (1999) : Sciences et théologie. Les figures d'un dialogue, Namur, Presses universitaires de Namur. 
MATHIEU S. (2011) : "Ce qu'ils en disent : la perception de la théorie de l'évolution par des élèves de collège et lycée ", in PORTIER P., VEUILLE M., WILLAIME J.-P. (dir.), Théorie de l'évolution et religions, Paris, Riveneuve éditions, p. 225-238.

MINOIS G. (1990) : L'Église et la science. Histoire d'un malentendu, Paris, Fayard.

RASI H. (2003) : "La foi, la raison et le chrétien cultivé », Dialogue, n 15(3), p. 5-9.

URVOY D. (2006) : Histoire de la pensée arabe et islamique, Paris, Seuil.

WOLFS J.-L. (2013): Sciences, religions et identités culturelles. Quels enjeux pour l'éducation?, Bruxelles, De Boeck.

WOLFS J.-L., DELHAYE C. (2016) : "Convictions religieuses et adoption ou nonadoption d'une conception sécularisée de la science. Enquête réalisée auprès d'élèves de terminale en Belgique francophone ", Revue de didactique des sciences religieuses, $\mathrm{n}^{\circ} 2$, p. 14-29. 